\title{
AC Magnetic Susceptibility of Ferrofluids Exposed to an External Electric Field
}

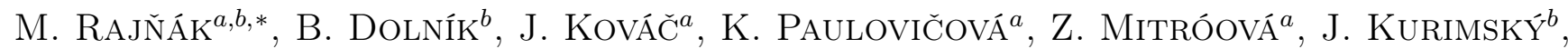 \\ R. Cimbala $^{b}$, P. KopČAnskÝ ${ }^{a}$ And M. TimkO ${ }^{a}$ \\ ${ }^{a}$ Institute of Experimental Physics, SAS, Watsonova 47, 04001 Košice, Slovakia \\ ${ }^{b}$ Faculty of Electrical Engineering and Informatics, Technical University of Košice, Letná 9, 04200 Košice, Slovakia
}

\begin{abstract}
It is known that ferrofluid superparamagnetic nanoparticles response to external magnetic fields, often resulting in the formation of elongated clusters along the field. This has a notable impact on dielectric properties of ferrofluids. Here we report on indications of a contrary effect when the magnetic susceptibility of ferrofluids based on transformer oil is influenced by an external electric field. This effect is associated with structural changes in the ferrofluids induced by the external electric fields. Particularly, we focus on a steady state electric field effect, which gives rise to forces acting on the magnetite nanoparticles, leading to the formation of aggregates. In this condition we have measured the ferrofluid AC magnetic susceptibility in parallel and perpendicular configuration of magnetic and electric fields at room temperature. The measurements in both configurations yielded a noticeable decrease in the real susceptibility values with increasing electric field intensity. The result is believed to be caused by the reduction in the total magnetic moment of the ferrofluid. This can be a consequence of the superspin interactions in the aggregates, minimizing the aggregate's energy. Finally, we highlight the necessity of NMR and small angle scattering of polarized neutrons investigations in order to obtain exact information on the magnetic structure induced by the electric forces.
\end{abstract}

DOI: 10.12693/APhysPolA.131.887

PACS/topics: 47.65.Cb, 75.75.Fk, 75.78.-n, 83.60.Np

\section{Introduction}

Ferrofluids are stable colloidal suspensions of superparamagnetic nanoparticles (SNP) in a base liquid [1]. During the last decades, they have been studied, especially because of their potentially large number of applications in different fields such as magnetism, optics, rheology, biophysics, medicine, and thermodynamics [2]. In the field of dielectrics and electrical engineering, ferrofluids based on transformer oil have been investigated as alternative insulating and cooling fluids for high voltage electrical equipment, e.g. power transformers. Many studies showed that these ferrofluids withstand higher voltages than pure transformer oils, the property often presented as a paradox $[3,4]$. In this context, a theoretical modelling has indicated that nanoparticles in transformer oil can act as free charge scavengers which slow down the streamer velocity leading to dielectric breakdown [5]. Therefore, the mobility of SNP also plays an important role in reducing the streamer velocity. For this reason, one can assume that knowledge about interactions and structural behavior of SNP in electric fields are of fundamental importance in the comprehension of the paradoxical breakdown mechanism.

Recently, it was deduced from dielectric measurements that SNP can form aggregates in electric fields [6]. Moreover, electric field induced formation of anisotropic struc-

*corresponding author; e-mail: rajnak@saske.sk tures greater than $300 \mathrm{~nm}$ was proved by small angle neutron scattering [7]. Thus, an open question has been raised regarding a relation between the electric field induced structural changes and magnetic properties of ferrofluids based on transformer oil. Can external electric forces acting on ferrofluids stop the superparamagnetic moment fluctuations in SNP and give rise to magnetic dipole-dipole interactions, forming so magnetic structures? An exact answer to this question could shed light on the peculiar breakdown mechanism, as the magnetic structures generating local stray fields could change the velocity direction of migrating charges during the streamer formation (local Lorentz forces). The question is equally interesting from a fundamental point of view, realizing that electromagnetic coupling in superparamagnetic liquid dispersions has not received much attention yet.

In this study we report on the first attempts to detect the ferrofluid magnetic changes due to external electric forces by measuring AC magnetic susceptibility of a sample exposed to various values of electric field intensity.

\section{Materials and methods}

Magnetite $\left(\mathrm{Fe}_{3} \mathrm{O}_{4}\right)$ SNP coated with oleic acid in transformer oil Mogul were synthesized by chemical coprecipitation [8]. Average particle size of $10 \mathrm{~nm}$ was found by a low voltage transmission electron microscope. The room temperature value of saturation magnetization of the studied ferrofluid is $29.6 \mathrm{kA} \mathrm{m}^{-1}$ corresponding to the magnetic volume fraction of $6.6 \%$. The sample exhibits the superparamagnetic behavior at room tempera- 
ture when the magnetization curve shows no hysteresis (Fig. 1). The blocked regime of the SNP at low temperature is reflected in the pronounced magnetic hysteresis. Temperature dependent ferrofluid magnetization measured under zero field cooled (ZFC) and field cooled (FC) conditions is depicted in Fig. 2. Both, ZFC and FC curves display the usual features found in superparamagnetic systems [9]. The arrow points to the area of blocking temperatures. The high temperature $\mathrm{ZFC}$ maximum is associated with a phase transition from frozen to liquid state of the oil (pour point). These measurements were performed by a SQUID magnetometer Quantum Design MPMS XL5.

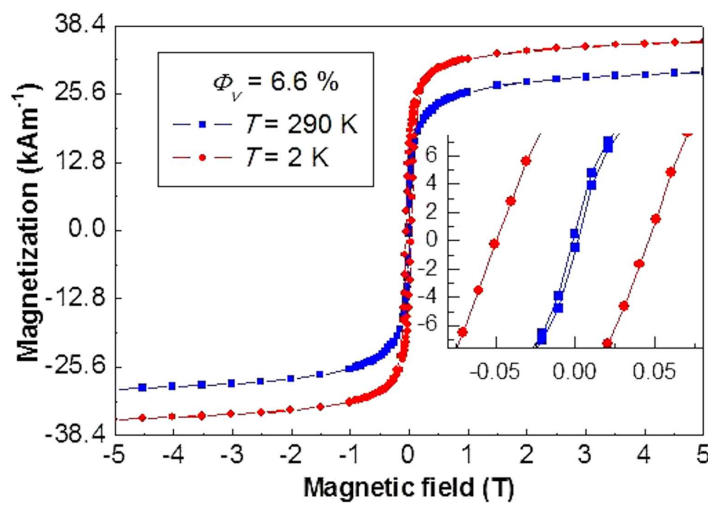

Fig. 1. Magnetization curves of the studied ferrofluid.

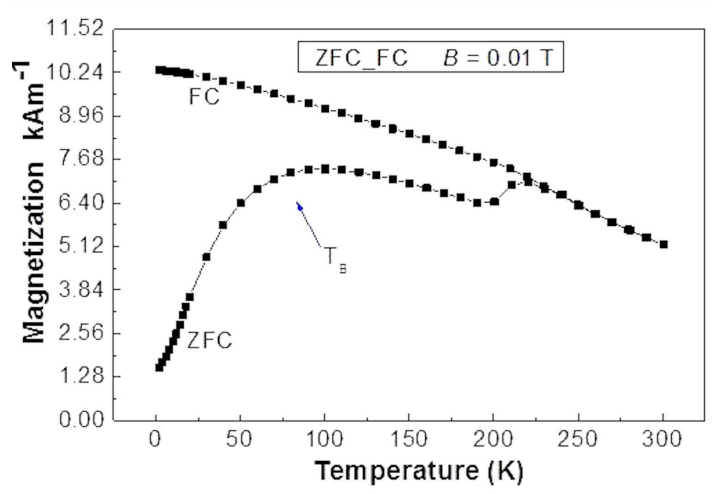

Fig. 2. ZFC and FC curves of the studied ferrofluid. The DC magnetic field was set to $0.01 \mathrm{~T}$.

In order to detect the AC susceptibility we used an AC susceptometer DynoMag, Imego with resolution in volume susceptibility $4 \times 10^{-7}$. The excitation field amplitude was $0.5 \mathrm{mT}$. The sample was measured in a glass vial equipped with two copper bar electrodes connected to a power supply. The field distribution in the vial was simulated by the finite element method magnetics (FEMM 4.2) using the triangular element mesh and the iterative conjugate gradient solver (Fig. 3). The dimensions of the automatically generated mesh were reduced in the near vial region by a factor of 100 . After measuring the susceptibility in a zero electric field, the sample was measured under the applied direct current (DC) voltage, ranging from $300 \mathrm{~V}$ to $2400 \mathrm{~V}$. The constant voltage was acting on the sample during each particular measurement that was performed in the frequency range from $1 \mathrm{~Hz}$ to $250 \mathrm{kHz}$. One measurement took $17 \mathrm{~min}$. The experiment was carried out at room temperature.

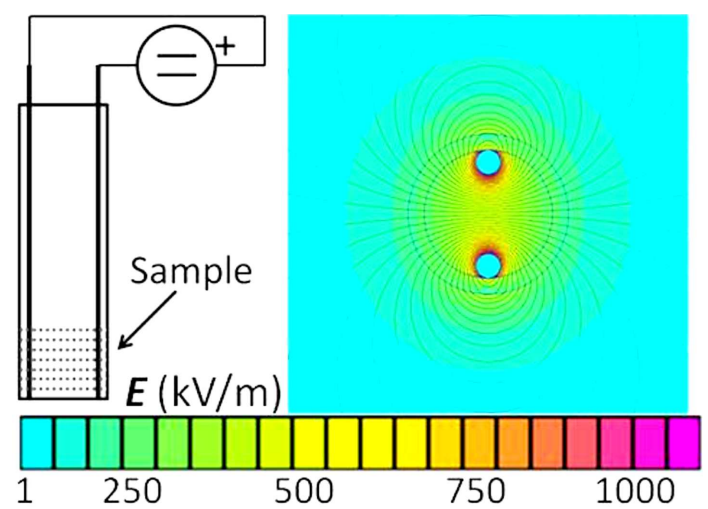

Fig. 3. (left) Schematic representation of the sample holder with the electrical circuit. The inner diameter of the vial is $6.5 \mathrm{~mm}$. The distance between the electrodes is $4 \mathrm{~mm}$. (right) Simulated equipotential field lines and the field intensity distribution (color scale) in the cross-section of the vial and electrodes with the potential difference of $2.1 \mathrm{kV}$.

\section{Results and discussion}

Taking into account the superparamagnetic state and the mean SNP diameter of $10 \mathrm{~nm}$, it can be determined that the internal moment reversal (the Néel relaxation) [9] is preferred to respond the magnetic field and is fast enough to follow the applied field changes. Hence, its relaxation maximum is expected to appear above the high frequency limit. However, moderately decreasing real susceptibility with increasing frequency reflects the presence of greater particles and the related size distribution (Fig. 4).

It was found that the increasing applied voltage causes a noticeable drop in the real susceptibility spectrum, but does not change its shape. This behavior is clearly seen in the selected lower frequency region (Fig. 4). The decreasing susceptibility reflects some reduction in the total magnetic moment of the ferrofluid. That reduction originates in the formation of particle aggregates induced by the applied DC electric field. As we confirmed in Ref. [7], DC electric field generated between two bar electrodes gives rise to formation of particle aggregates greater than $300 \mathrm{~nm}$. One can assume that in such an aggregate the SNP superspins will interact in a way leading to the minimization of its total energy. As the susceptibility spectrum has not been changed even under the maximal applied voltage, the superspin interactions apparently do not cause the aggregates to respond 


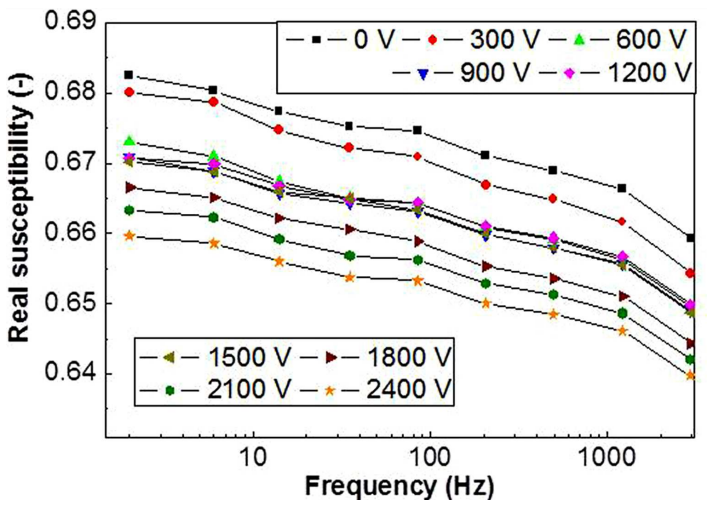

Fig. 4. Real susceptibility spectrum in dependence on the applied electrical voltage.

the AC magnetic field by Brownian relaxation, which would appear in the low frequency region. Instead, magnetic alignment arises in the aggregates, which introduces some optimal states with compensated SNP superspins (e.g. antiferromagnetic-like alignment). On the other hand, the critical diameter of magnetite $\left(\mathrm{Fe}_{3} \mathrm{O}_{4}\right)$ particles below which the single domain state is preferable is $98 \mathrm{~nm}$ [9]. Therefore, in greater aggregates of interacting SNP a domain structure can be introduced. Any kind of superspin compensation in the aggregates will finally lead to the decrease in the total ferrofluid magnetic susceptibility. In this case, only individual SNP and uncompensated spins in the aggregates contribute to the total ferrofluid response in the low magnetic fields. This behavior thus resembles the well known decrease in ferrofluid susceptibility with decreasing magnetic particle concentration in a base liquid.

Considering the SNP interactions in the aggregates, one should also bear in mind an impact on the magnetic anisotropy energy. Any formation of magnetic structure in the aggregates certainly increases that energy. Consequently, higher magnetic fields are required to deflect the compensated superspins.

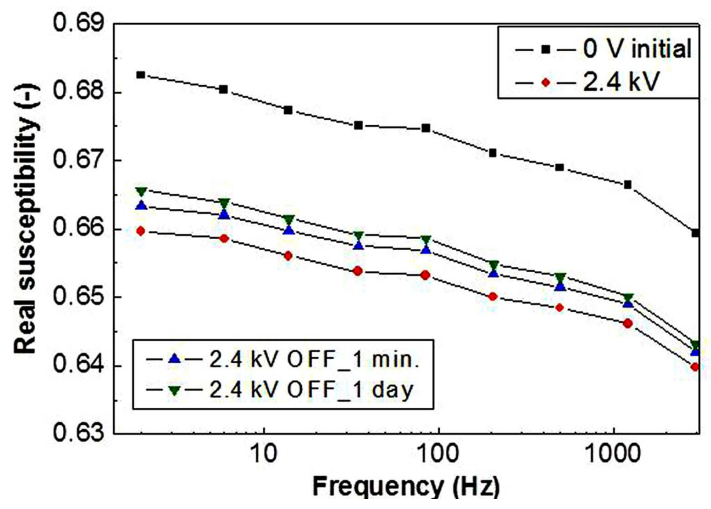

Fig. 5. Real susceptibility spectrum behavior after switching the DC voltage off.
Finally, when the applied voltage is switched off, the susceptibility spectrum tends to return back to higher values (Fig. 5). This is associated with the thermally stimulated destruction of the aggregates. However, as the original susceptibility level has not been achieved even after a day of rest, some aggregates still persist. The described behavior has been observed also in the case when the electric field direction was applied in parallel to the excitation AC magnetic field.

\section{Conclusions}

The reported results provide solid evidence of electric field effect on the AC magnetic susceptibility of transformer oil-based ferrofluid. The effect can be associated with SNP dipole-dipole interactions forming a magnetic structure. To prove and find out the specific magnetic structure, methods like NMR and small angle scattering of polarized neutrons (SANSPOL) should be applied. Any magnetic structure formation can have remarkable impact on the other important properties and phenomena in the ferrofluid, like dielectric breakdown and thermomagnetic convection.

\section{Acknowledgments}

This work was supported by Slovak Academy of Sciences and Ministry of Education: VEGA 2/0016/17, $1 / 0311 / 15,2 / 0141 / 16,1 / 0377 / 16$, Ministry of Education Agency for structural funds of EU, Project No. 26220120046, 26220120055, 26220120033 and APVV-150438. We acknowledge Dr. Elena Alina Taculescu-Moaca for preparing the investigated ferrofluid.

\section{References}

[1] R.E. Rosensweig, Ferrohydrodynamics, Courier Dover Publ., New York 1997.

[2] C. Scherer, A.M. Figueiredo, Braz. J. Phys.35, No. 3A (2005).

[3] V. Segal, A. Hjortsberg, A. Rabinovich, D. Nattrass, K. Raj, in: Conference record of the 1998 IEEE Int. Symp. on Electrical Insulation, 1998, p. 2.

[4] J.Ch. Lee, H.S. Seo, Y.J. Kim, Int. J. Therm. Sci. 62, 29 (2012).

[5] J.G. Hwang, M. Zahn, F.M. O'Sullivan, L.A.A. Pettersson, O. Hjortstam, R. Liu, J. Appl. Phys. 107, (2010).

[6] M. Rajnak, J. Kurimsky, B. Dolnik, P. Kopcansky, N. Tomasovicova, E.A. Taculescu-Moaca, M. Timko, Phys. Rev. E 90, 032310 (2014).

[7] M. Rajnak, V.I. Petrenko, A.M. Avdeev, I.O. Ivankov, A. Feoktystov, B. Dolnik, J. Kurimsky, P. Kopcansky, M. Timko, Appl. Phys. Lett. 107, 073108 (2015).

[8] L. Vékás, D. Bica, M.V. Avdeev, China Particuol. 5, 43 (2007).

[9] O. Petracic, Superlatt. Microstruct. 47, 569 (2010). 